\title{
A quantum-based approach to predict primary radiation damage in polymeric networks
}

\author{
Matthew P. Kroonblawd, ${ }^{*} \dagger$ Nir Goldman, ${ }^{\dagger, \dagger}$ Amitesh Maiti, ${ }^{\dagger}$ and James P. \\ Lewicki $^{\dagger}$ \\ $\dagger$ †hysical and Life Sciences Directorate, Lawrence Livermore National Laboratory, \\ Livermore, CA 94550, United States \\ $\ddagger$ Department of Chemical Engineering, University of California, Davis, California 95616, \\ United States \\ E-mail: kroonblawd1@|lnl.gov
}




\begin{abstract}
Initial atomistic-level radiation damage in chemically reactive materials is thought to induce reaction cascades that can result in undesirable degradation of macroscale properties. Ensembles of quantum-based molecular dynamics (QMD) simulations can accurately predict these cascades, but extracting chemical insights from the many underlying trajectories is a labor-intensive process that can require substantial a priori intuition. We develop here a general and automated graph-based approach to extract all chemically distinct structures sampled in QMD simulations and apply our approach to predict primary radiation damage of polydimethylsiloxane (PDMS), the main constituent of silicones. A post-processing protocol is developed to identify underlying polymer backbone structures as connected components in QMD trajectories. These backbones form a repository of radiation-damaged structures. A scheme for extracting and updating a library of isomorphically distinct structures is proposed to identify the spanning set and aid chemical interpretation of the repository. The analyses are applied to ensembles of cascade QMD simulations in which the four element types in PDMS are selectively excited in primary knock-on atom events. Our approach reveals a much higher degree of combinatorial complexity in this system than was inferred through radiolysis experiments. Probabilities are extracted for radiation-induced network changes including formation of branch points, carbon linkages, cycles, bond scissions, and carbon uptake into the Si-O siloxane backbone network. The general analysis framework presented here is readily extendable to modeling chemical degradation of other polymers and molecular materials and provides a basis for future quantum-informed multiscale modeling of radiation damage.
\end{abstract}

\title{
1 Introduction
}

Purposeful exposure of materials to ionizing radiation is a reliable means of sterilization ${ }^{1-3}$ and is a common approach taken in accelerated aging experiments. ${ }^{4-6}$ In molecular materials, radiation exposure can induce atomic-level chemical reactions that alter or degrade many 
properties, including those which impart functional characteristics that must meet tolerance specifications. Chemical degradation of polymer materials is thought to arise from networkaltering reactions such as chain scissions and formation of cross links, which can lead to macroscopic mechanical changes including permanent set, embrittlement, or breaking under load. ${ }^{2,7,8}$ Experimental diagnostics that probe chemical degradation are often indirect and capture highly integrated responses from which it can be hard to infer fundamental chemical events, for instance measuring viscosity, gelation, stress-strain responses, vibrational spectra, or evolved off-gassing products. ${ }^{9-15}$ Nuclear magnetic and electron paramagnetic resonance experiments provide some of the most direct measures of time- and dose-dependent chemistry and network changes in irradiated polymers. ${ }^{12,13,16-19}$ Atomistic modeling techniques such as reactive molecular dynamics (MD) can provide insight into underlying network-altering chemistry in polymers, ${ }^{15,20-28}$ but upscaling these insights to inform models for macroscale responses is challenging. A desirable foundation for multiscale modeling of radiation damage in polymers would incorporate accurate high throughput sampling of atomic-scale chemistry and an automated scheme for extracting statistics on changes to the network.

Radiation damage can be separated into two classes based on process timescales. ${ }^{29}$ Primary damage is due to initial ballistic atomic motions induced by radiation-matter coupling that occur on fs to ps time scales. Specific characteristics of the damage depend on the material class, but include for instance point crystal defects such as vacancies or interstitials, ${ }^{30-34}$ local melting or amorphization, ${ }^{35-37}$ and in the case of chemically reactive materials, the breaking and formation of bonds. ${ }^{11,25,26,38,39}$ A convenient time origin for local primary damage is taken to be the primary knock-on atom (PKA) event, in which the first atom recoils from the interaction and begins to move with a large kinetic energy. ${ }^{30}$ To induce damage, the energy associated with a PKA event must be much larger than the local binding energy, and is minimally a few eV for many types of materials. This large input energy drastically raises the local "temperature" by thousands of Kelvin or more. ${ }^{32}$ As ballistic motions cease, thermal conduction sets in and the damaged region returns to thermal equilibrium with 
the surrounding material. Following this, secondary damage arises over longer timescales as defects produced in primary damage anneal, migrate, and interact with each other and the pristine material through thermally activated processes.

Molecular dynamics is particularly well suited to study primary radiation damage on its natural time and length scales. ${ }^{25,26,30-33}$ Given an appropriate description of the potential energy, one specifies a nonequilibrium starting condition that is representative of the PKA event (typically assigning an atom a large velocity) and then integrates a constant-volume constant-energy $(N V E)$ MD trajectory to predict the cascade of events that ensue. A PKA simulation approach allows for the exploration of chemical phase space without explicit modeling of absorption cross-sections, and subsequent electronic excitations and electron-phonon couplings. As these nonequilibrium cascade processes can be highly path-dependent, an ensemble methodology is usually adopted for better sampling in which dozens or hundreds of independent simulations are performed starting from microscopically different but macroscopically equivalent initial conditions. The main practical difficulty of this approach lies in analyzing the outcomes of hundreds of simulations. Many, if not most, MD studies of radiation damage have focused on materials with simple compositions and structures (e.g., metals) for which there has been considerable investment to develop order parameters or descriptors that are sensitive to crystal defects. ${ }^{40-43}$ In chemically reactive molecular materials, this analysis is especially cumbersome due to a large chemical phase space involving many intermediates, transition states, and complicated radicals.

Graphs, which at their most basic are simply a collection of nodes connected by edges, have long formed a basis for mathematical chemistry and machine-recognition of chemical structure. ${ }^{44}$ Covalent connectivity in molecules is conveniently and intuitively captured by graphs, with atom centers as nodes and chemical bonds as edges. Assigning attributes to nodes and edges adds further flexibility to graphs, allowing for descriptions of element types, charge states, chirality centers, and bond multiplicity, among other properties typically associated with atoms and bonds in molecules. Using graphs as a chemical descriptor 
avoids common pitfalls with degeneracy, where multiple chemically "distinct" structures are rendered indistinguishable. Simple descriptors such as chemical formulas are often highly degenerate; for instance, $\mathrm{C}_{4} \mathrm{H}_{10}$ can refer to the isomers butane or 2-methylpropane. Modern general-purpose graph analysis software tools ${ }^{45,46}$ and metrics provide powerful and portable engines for working directly with graphs and facilitate developing tailored ${ }^{47-52}$ algorithms and analyses for molecular systems.

Accuracy of the potential energy surface for MD simulations of PKA events is a key consideration for atomistic modeling of radiation damage. Density functional theory ${ }^{53}$ (DFT) is the standard for accurate quantum-based MD (QMD) predictions of chemistry at extreme conditions. However, the computational expense of DFT usually precludes running many independent simulations that are necessary to adequately sample nonequilibrium processes in chemically reactive materials. Density functional tight-binding ${ }^{54,55}$ (DFTB) is a semiempirical approach derived from DFT that retains much of its accuracy while reducing the computational expense by orders of magnitude. With DFTB, it is practical to obtain many ns of QMD trajectory through ensemble sampling, which is critical for developing quantuminformed coarse-grained models through statistical sampling of microstates connected to macroscopic observables. ${ }^{52,56-60}$ This makes the DFTB method a good choice for placing studies of radiation damage in reactive molecular materials on a firmer "quantum" basis.

We develop here a general, automated, graph-based approach to analyze ensembles of reactive QMD simulations run with DFTB and extract all chemically distinct structures. We apply our approach to predict primary radiation damage of polydimethylsiloxane (PDMS), the main constituent of silicones, which are ubiquitous in commercial, industrial, and medical applications. ${ }^{61}$ A post-processing protocol is developed to identify underlying polymer backbone structures as connected components in QMD trajectories. These backbones form a repository of radiation-damaged structures. A scheme for extracting and updating a library of isomorphically distinct structures is proposed to identify the spanning set and aid chemical interpretation of the repository. The analyses are applied to ensembles of cascade 
QMD simulations in which the four element types in PDMS are selectively excited in PKA events. Probabilities are extracted for radiation-induced network changes including formation of branch points, carbon linkages, cycles, bond scissions, and carbon uptake into the Si-O siloxane backbone network. Highly detailed motifs are identified among the spanning set of backbone structures, indicative of chemistry that is decidedly more complicated and varied than simple reaction schemes for chain scissioning and cross linking.

\section{Methods}

\subsection{QMD Simulations}

High-throughput QMD simulations were performed using the self-consistent charge DFTB method $^{54,55}$ as implemented in the DFTB + code. $^{62}$ The DFTB total energy is derived from an expansion of the Kohn-Sham energy ${ }^{53}$ about a reference electronic density to second order in charge fluctuations and is evaluated as

$$
E_{\mathrm{DFTB}}=E_{\mathrm{BS}}+E_{\mathrm{Coul}}+E_{\mathrm{Rep}}+E_{\text {Disp }}
$$

Here $E_{\mathrm{BS}}$ is the electronic band structure energy evaluated in a tight-binding framework ${ }^{63}$ with a minimal atomic orbital basis, $E_{\text {Coul }}$ captures electrostatic interactions between fluctuating partial charges, and $E_{\text {Rep }}$ and $E_{\text {Disp }}$ are empirical repulsion and dispersion terms. We used the DFTB parameter set pbc-0-3 (available at http://www.dftb.org), a typical offthe-shelf parameter set for silicon-containing systems that we previously applied to study phenyl excitations in phenylated siloxane copolymers ${ }^{28}$ and the mechanochemical susceptibility of strained siloxane chains. ${ }^{50}$ Universal force field dispersion terms ${ }^{64}$ were used for $E_{\text {Disp }}$. The electronic structure was evaluated without spin polarization at the $\Gamma$-point only using Fermi-Dirac thermal smearing ${ }^{65}$ and a self-consistent field (SCF) accuracy threshold of $2.7 \times 10^{-5} \mathrm{eV}\left(10^{-6} \mathrm{H}\right)$. Specific thermal smearing energies (temperatures) are noted below. 
All simulations were performed using extended Lagrangian Born-Oppenheimer dynamics (XL-BOMD). ${ }^{66-69}$

A model amorphous PDMS system was constructed that consisted of a cubic 3D-periodic cell that contained four chains of dodecamethylpentasiloxane (DMPS), a methyl-terminated PDMS backbone with five silicon centers. Each DMPS chain has 57 atoms, yielding a cell with 228. A multistep procedure was used to bring the system to a crystal-like density ${ }^{70,71}$ of $1.2 \mathrm{~g} \cdot \mathrm{cm}^{-3}$ and to equilibrate it at $300 \mathrm{~K}$ prior to simulations to predict primary radiation damage. QMD trajectories for system preparation were integrated using the LAMMPS code $^{72}$ with forces and stresses evaluated by DFTB + . The temperature was set to $300 \mathrm{~K}$ through a Nosé-Hoover-style thermostat, ${ }^{73,74}$ the electron temperature was set equal to the instantaneous ionic temperature, and the XL-BOMD dynamics were integrated with a 0.2 fs time step and four SCF cycles per step. First, the four DMPS chains were placed in a cubic cell with side length $20 \AA$. This cell was then isotropically compressed to $1.2 \mathrm{~g} \cdot \mathrm{cm}^{-3}$ by reducing the cell length to $12.867 \AA$ at a constant rate of $1.0 \AA \cdot \mathrm{ps}^{-1}$. Following this, a 20 ps isothermal-isochoric $(N V T)$ simulation was performed and 100 configurations were extracted every 100 fs during the last 10 ps as independent starting points for simulations of radiation damage.

The ensuing reactions that follow from PKA events in PDMS were predicted using cascade QMD simulations. In a cascade simulation, the staring point is a PKA event in which a randomly selected atom in a thermal configuration is imparted with a large "recoil" kinetic energy through assignment of a random velocity of set magnitude. Recoil energies in the 10-100 eV range are typical in such simulations. We take a representative recoil energy to be $50 \mathrm{eV}$, which is $O(10)$ times greater than typical bond dissociation energies in PDMS and is similar in magnitude to ultraviolet radiation. Given the large chemical phase space that is accessible under such conditions, we sampled the initial reactions that lead to primary radiation damage through ensembles of statistically independent 10 ps long cascade simulations. Thermal relaxation due to conduction of the resulting heat spike in nm-sized regions 
is expected to be on the order of $10 \mathrm{~s}$ of ps or more for materials with a glass-like thermal conductivity $\left(\lambda<0.5 \mathrm{~W} \cdot \mathrm{m}^{-1} \cdot \mathrm{K}^{-1}\right)$ such as PDMS. ${ }^{75}$

A given ensemble had 100 independent simulations, each starting from one of the 100 independent starting configurations, and a single atom type ( $\mathrm{Si}, \mathrm{O}$, C, or H) was selected for PKA excitation; the single excited atom in a given simulation was chosen at random from among those of the chosen type. Four ensembles were considered, one for each atom type, leading to 400 independent trajectories and a total of $4 \mathrm{~ns}$ of combined QMD trajectory. All cascade QMD simulations were performed with the standalone DFTB + code using $N V E$ XL-BOMD dynamics. The electron temperature was set to $2000 \mathrm{~K}$, consistent with the system-average kinetic energy, and full SCF convergence was enforced each time step. A time step of 0.1 fs was used for simulations with recoil energy imparted to heavy atoms (Si, $\mathrm{O}$, and $\mathrm{C}$ ) and a time step of 0.05 fs was used for simulations with recoil imparted to $\mathrm{H}$ in order to ensure conservation of energy. Trajectory snapshots were recorded every 10 fs for subsequent chemical analysis.

\subsection{Structure Analysis}

Quantifying radiation damage at the atomic scale in chemically reactive molecular materials remains an open-ended problem. In polymers, chemical alterations to network structures through chain scissions and formation of cross-links are thought to be a primary driver for degradation of bulk mechanical properties. ${ }^{19}$ We develop here an automated graph-based structure recognition approach to quantify network damage predicted in reactive MD simulations. Our approach identifies all isomorphically unique backbones sampled in QMD trajectories and compiles a comprehensive library of graph structures that capture backbone topology for subsequent analysis. The state of backbones in the system can thus be assembled for any trajectory snapshot from the enumerated structures in the library.

The first stage in constructing a library of unique backbones is to post-process QMD trajectories to identify all underlying backbone structures. Our approach is summarized in 

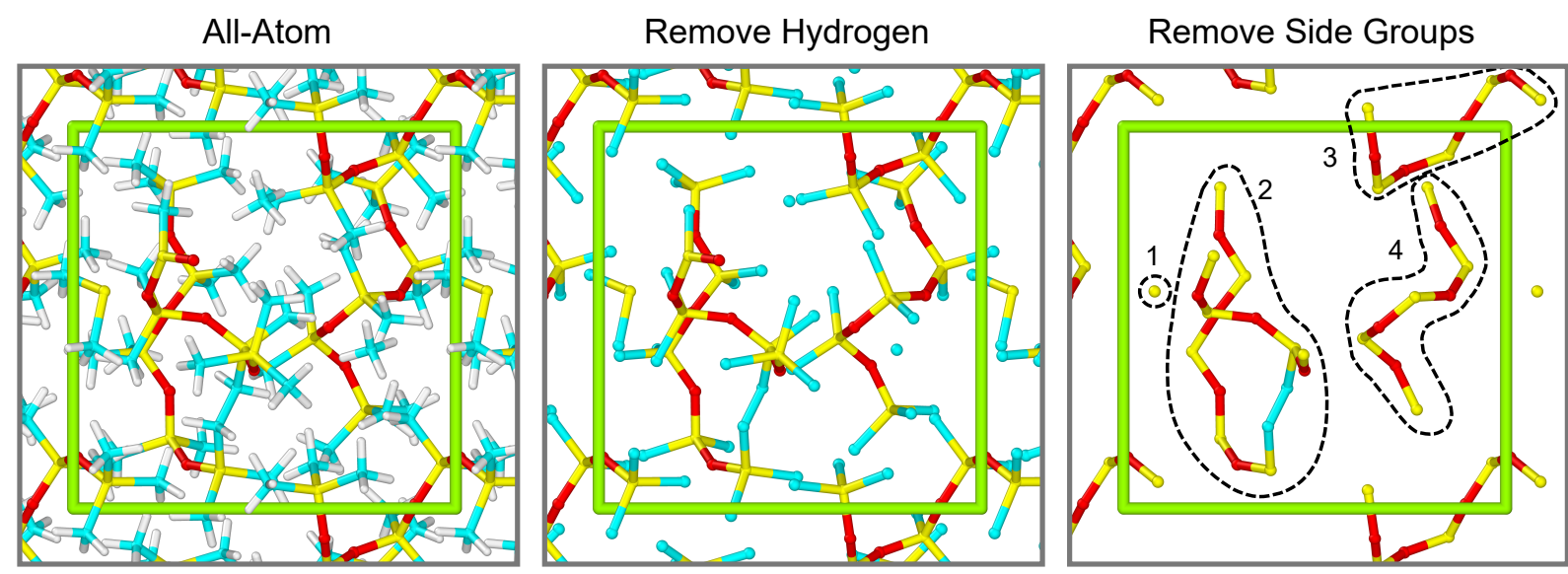

Figure 1: Procedure to identify network backbone structures. After removing hydrogen atoms and side groups, NetworkX is used to identify individual backbone structures as connected components. Atoms are colored yellow, red, cyan, and white for Si, O, C, and H atoms. The primary simulation cell is drawn with light green lines and truncated replications are shown to highlight the packing structure.

Table 1: Bonding criteria for automated structure recognition

\begin{tabular}{cc}
\hline \hline Interaction Type & Bonding Cutoff $(\AA)$ \\
\hline Si-Si & 2.2 \\
Si-O & 2.2 \\
Si-C & 2.2 \\
O-O & 1.8 \\
O-C & 1.8 \\
C-C & 1.8 \\
\hline \hline
\end{tabular}

Figure 1. First, hydrogen atoms are removed from the all-atom trajectory and covalent connectivity is assessed using a distance criterion. Two atoms were considered bonded if their separation distance fell within prescribed cutoffs taken to be slightly larger than typical equilibrium bond lengths (see Table 1). Then, side groups (e.g., methyls) were systematically identified and removed by deleting all atom centers that had one or fewer bonds. This results in a set of atoms and bonds that spans the backbones in the system. The opensource graph analysis software Network $\mathrm{X}^{46}$ was then used to identify the individual backbones as connected components. Taken over all the trajectories in an ensemble, this set of operations yields a repository of all sampled backbones, including many that are topologically indistinguishable. Simple metrics can be readily applied to the repository at this stage to extract 


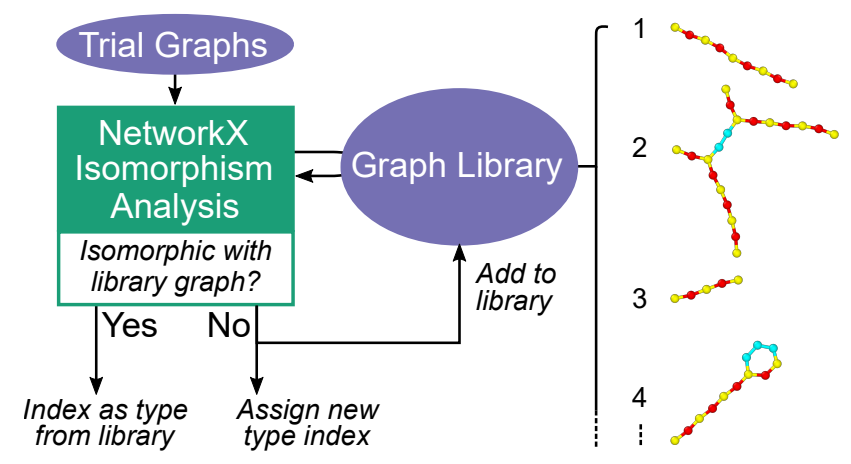

Figure 2: Schematic for building a library of isomorphic backbones sampled in QMD trajectories. Graph snapshots were rendered using the approach shown in Figure 3 with Si, O, and $\mathrm{C}$ atoms colored yellow, red, and cyan.

descriptors with limited chemical specificity, such as backbone size (atom count), elemental composition (chemical formulas), and counts of particular bond types.

The primary challenge in interpreting the chemical and mechanical significance of reactive trajectories is to identify the set of isomorphically unique (i.e., chemically and topologically identical) backbones that spans the full repository. In the second stage of the analysis, NetworkX is used to identify this spanning set as a library of graphs. Figure 2 shows a schematic of our approach. Here, NetworkX is used to identify whether a trial backbone from the repository is isomorphic to other structures in a library. The isomorphism check is performed using the the VF2 algorithm ${ }^{76}$ and considers backbones as graphs with a set of nodes (atoms) that have element type as an attribute and with unweighted, undirected edges (bonds) that do not distinguish bond multiplicity. Two graphs are considered isomorphic if there is at least one one-to-one function that maps the nodes from one graph to the nodes of the other while preserving the branch structure (i.e., an isomorphism). Adding element type as a node attribute adds a semantic criterion that requires the branch structure plus node attribute labeling to match for a mapping to be considered an isomorphism. Thus, all possible mappings must be checked to determine if any are isomorphisms and the two graphs therefore isomorphic or not. If a trial graph is not isomorphic to one in the library, it is automatically indexed and appended for future comparisons and analysis. Because determining isomorphism for general graphs is computationally demanding, we limit the 
number of such operations by only performing them for graph pairs that have identical numbers of each atom type and bond type.

Interpreting the identified graph library through rendering the underlying atomic coordinates is cumbersome as the backbone structures can have complex 3D foldings that make it challenging to pick out easy-to-visualize configurations by hand. We use an MD-inspired approach to automate the production of physically representative non-overlapping 2D plots of library graph structures to aid chemical interpretations. Stages of this procedure are shown in Figure 3. First, the graph for a backbone was used to generate a LAMMPS force field topology file for the structure that included all two-center bonds and three-center angles. Harmonic potentials were defined for the bonds, $U(r)=\frac{K_{r}}{2}\left(r-r_{0}\right)^{2}$, and angles, $U(\theta)=\frac{K_{\theta}}{2}\left(\theta-\theta_{0}\right)^{2}$, in which the potential parameters were set to $K_{r}=200 \mathrm{kcal} \cdot \mathrm{mol}^{-1} \cdot \AA^{-2}$, $r_{0}=1.5 \AA, K_{\theta}=100 \mathrm{kcal} \cdot \mathrm{mol}^{-1} \cdot \mathrm{rad}^{-2}$, and $\theta_{0}=170^{\circ}$. These values were chosen as reasonably physical constants that would allow for the generation of realistic backbone structures. An initial configuration was generated in which each atom coordinate was assigned a random value in $[0,1]$ and all these atoms were placed in the center of a cubic $3 \mathrm{D}$ periodic cell with side length $20 \AA$. The structure was "unfolded" in 3D through a series of alternating short NVT dynamics and optimization stages in which each atom was sequentially "unfixed" from its starting position and treated as flexible. At first, only one atom was allowed to move, and by the end of the sequence the entire structure was unfolded and largely non-overlapping. An optimization of the whole structure was performed with an additional purely repulsive truncated Lennard-Jones potential $\left(\epsilon=0.1 \mathrm{kcal} \cdot \mathrm{mol}^{-1}, \sigma=3 \AA, r_{\text {cut }}=3.367 \AA\right)$ to resolve any remaining branch overlaps. Finally, the structure was "flattened" to a quasi 2D representation through a 10 ps simulation in which the $z$-dimension of the cell was uniformly deformed to $2.2 \AA$. Snapshots of the structures thus obtained were rendered using OVITO. ${ }^{77}$ Structure preparation takes only seconds on a single CPU given the computational efficiency of the classical potential employed. We note that performing the initial unfolding in 3D proved critical as an explicitly 2D simulation approach frequently yielded irreconcilable 


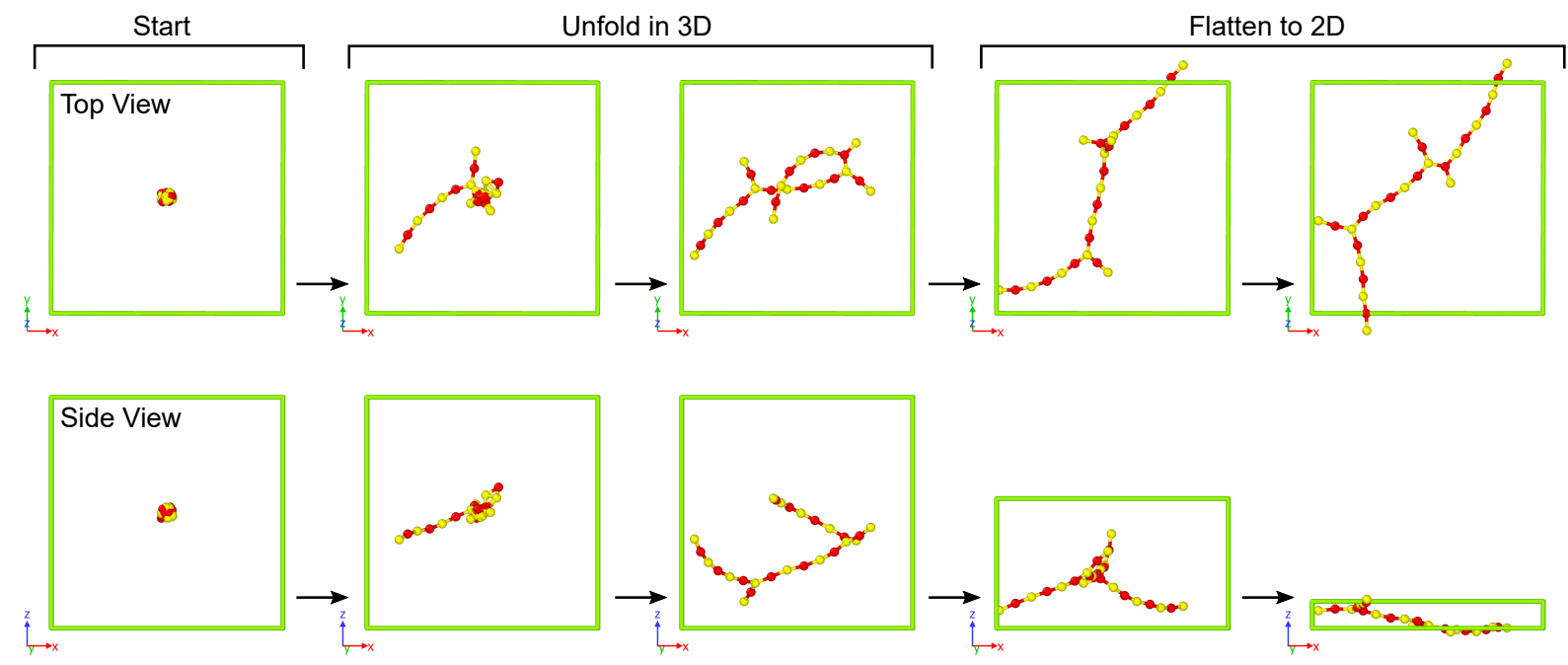

Figure 3: MD-inspired approach to automatically generate 2D plots of complicated backbone graph structures. The backbone is "unfolded" in 3D and "flattened" to 2D through a sequence of dynamics trajectories and structure optimizations under an applied generic force field.

branch overlaps. In following this recipe we were able to generate representative structures for each library index, allowing for straightforward chemical interpretation of each unique graph. This approach was only used to generate plots of backbone graph structures, and the generic force field was not employed in any other analysis.

While the above structure analysis approach is applied here to polymer backbones, we note that the basic aspects are quite general and can easily be applied to unprocessed allatom data to identify unique molecular structures. The main complication of working with all-atom data is the significant increase in dimensionality of the chemical phase space and a corresponding increase in typical graph size, which increases the cost of isomophism checks. Despite the computational expense, this approach has advantages over more efficient stringbased descriptors such as SMILES ${ }^{78}$ or canonical SMILES ${ }^{79}$ that are targeted solutions for managing and manipulating databases of stable compounds. One of these is that working with graphs directly yields an intuitive and unambiguous canonical representation for each structure. The other, more significant advantage is that it naturally accommodates reactive intermediates, transition states, and complicated radicals that are frequently encountered in reactive MD chemistry studies. 


\section{Results and Discussion}

\subsection{Time-Dependent Response}

Primary radiation damage in PDMS was predicted using ensembles of QMD cascade simulations following the approach given in Section 2.1. We focus first on high-level characteristics of the time-dependent response following a PKA event. Ensemble-averaged time histories were computed for each excitation type that track changes in simple backbone descriptors identified using the approach summarized in Figure 1.

Important characteristics that influence the mechanical properties of a polymer network include the number of backbones and their connections (e.g., number of connected components and bonds) and changes in chemical composition. Figure 4(a) shows the ensembleaverage change in the number of backbone bonds between silicon, oxygen, and carbon atoms. In cases where the side group atoms are excited $(\mathrm{C}$ and $\mathrm{H})$, there is a net formation of new connections. With carbon excitations, these new connections are formed quickly within the first 2 ps following the PKA event. The growth is more gradual in the case of hydrogen excitations. In contrast, direct excitations of the backbone ( $\mathrm{Si}$ and $\mathrm{O}$ ) initially lead to a loss of connectivity. This is not unexpected, as these PKA events lead to backbone scission reactions as the first chemical event. Over time, subsequent reactions produce new backbone connections, but the total number of these new connections does not compensate for those lost in the initial event. A probabilistic interpretation of the results at $10 \mathrm{ps}$ indicates that a single radiation event will form a new connection $97 \%$ (or 91\%) of the time when carbon (or hydrogen) atoms are excited. Perhaps surprisingly, initial scissions from excitation of silicon largely anneal. There is a $70 \%$ chance for a persistent loss of one network connection in the case of oxygen excitations.

Carbon uptake into the network is captured in Figure 4(b). Specific ways in which carbon becomes incorporated in the network will be assessed in detail in Section 3.2. Two features are evident from the time history. One is that all four excitation types can lead to new 

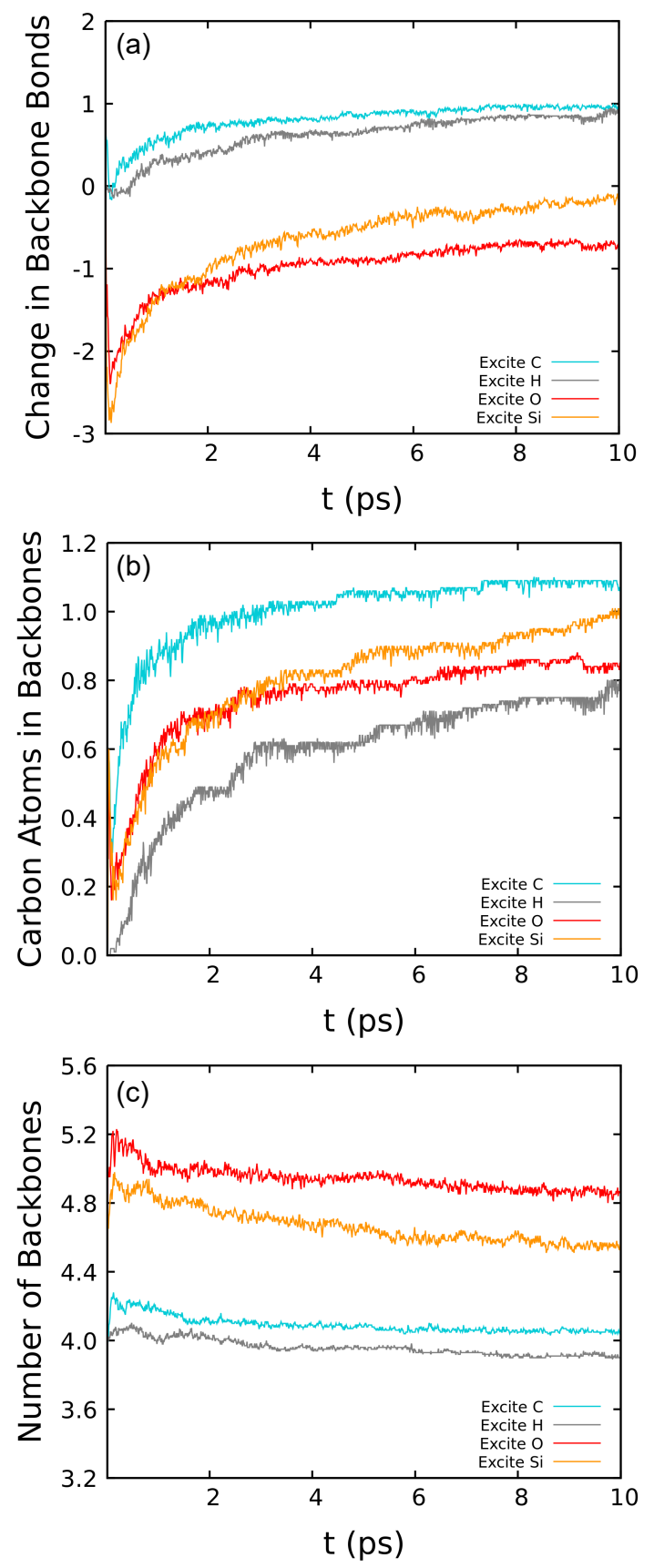

Figure 4: Ensemble-averaged time histories for each excitation type showing (a) the number of backbone bonds, (b) the number of carbon atoms incorporated into backbones, and (c) the number of individual backbones in the system.

backbone bonds with carbon. The other is that most of the reactions responsible for this incorporation occur within the first 2 ps. Apparent minima at very early times $(<1 \mathrm{ps})$ in the silicon and oxygen curves likely result from backbone bonding being determined with 
only a distance (not lifetime) criterion combined with the highly ballistic nature of the initial PKA event. Exciting carbon atoms directly is $\approx 35 \%$ more effective at incorporating carbon into the network as exciting hydrogen, which is the most ineffective of the four types.

The influence of scission reactions is immediately apparent in the ensemble-average backbone counts for the system shown in Figure 4(c). In cases where the backbone is excited directly ( $\mathrm{Si}$ and $\mathrm{O})$, one has a very rapid $(<1 \mathrm{ps})$ increase from the four initial backbones to approximately five (e.g., a single scission event). Exciting carbon atoms also produces a small initial increase in the number of backbones or chain scission events. For all four excitation types, there is a slow and steady decrease in the total backbone count over time due to various cross-linking and fragment-recombination reactions. Comparison of the three panels shows that while the total number of connections largely recovers for silicon excitations, these newly formed connections do not necessarily imply a complete return to the starting number of backbones. Rather, once scissioned, many backbones go on to react with side groups and increase in carbon content without forming cross-links between backbones. Only in the case of hydrogen excitations is there a net decrease in the number of backbones relative to the starting point, implying some possibility of the formation of cross-linked structures overall.

Complete time histories for the evolution of backbone sizes averaged over all simulations in each ensemble are captured in Figure 5. It is clear even from this simple descriptor that the topological evolution of the system is quite complicated. While each system starts with four backbones, each containing nine atoms, the PKA event almost instantaneously $(<1$ ps) produces a wide range of different backbone sizes from only 1-2 atoms up to roughly twice the starting size ( $\approx 18$ atoms). Direct excitation of the backbone silicon and oxygen atoms almost instantly produces many small backbones with an approximately uniform distribution. This is due to chain scissions being among the first chemical reactions in the cascade and the selection of specific recoil atoms at random. Fewer small backbones are generated when side group atoms $(\mathrm{C}$ and $\mathrm{H})$ are excited, as chain scissions occur later in the reaction cascade if at all. In a few rare cases, after 5 ps, large backbones start to form that reach sizes roughly 

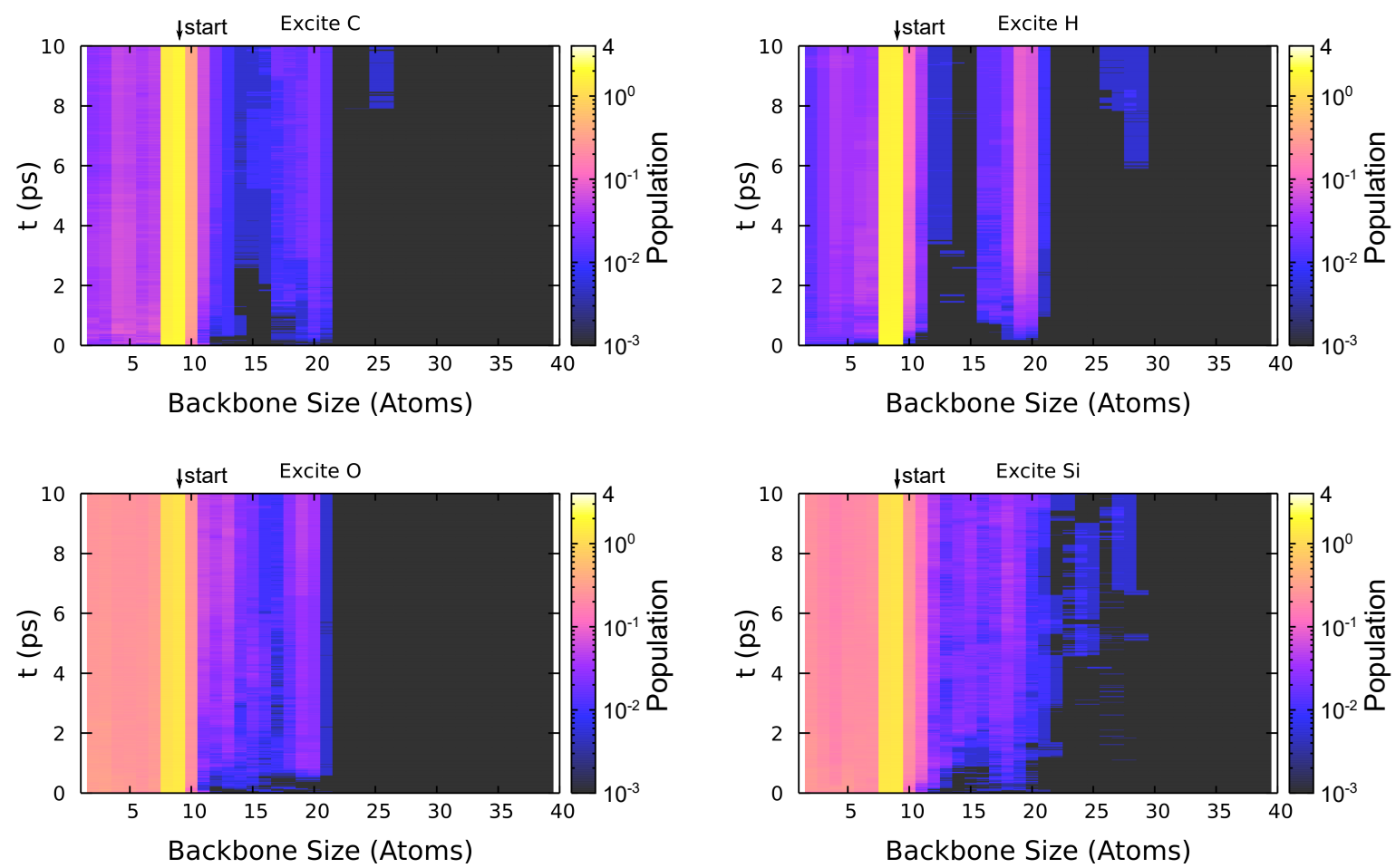

Figure 5: Heat maps showing the ensemble-average time evolution of backbone sizes measured in terms of number of backbone atoms for each of the four excitation types.

triple that of the starting size ( $\approx 27$ atoms). The formation of very large backbones is a comparatively slow and low-yield process.

\subsection{Network Analysis}

Simple descriptors such as backbone size, bond count, or carbon content cannot capture the underling complexity of the network changes that arise from reaction cascades. We applied our graph isomorphism analysis summarized in Figure 2 to the resulting products from the QMD simulations. The complete library of backbone structures thus identified is shown in Figure 6. There were a total of 1731 individual backbones, of which our approach found 160 that were unique.

There is surprising diversity among the various unique backbone structures, but some general chemical trends are apparent from our graph-based analysis. The first is that back- 


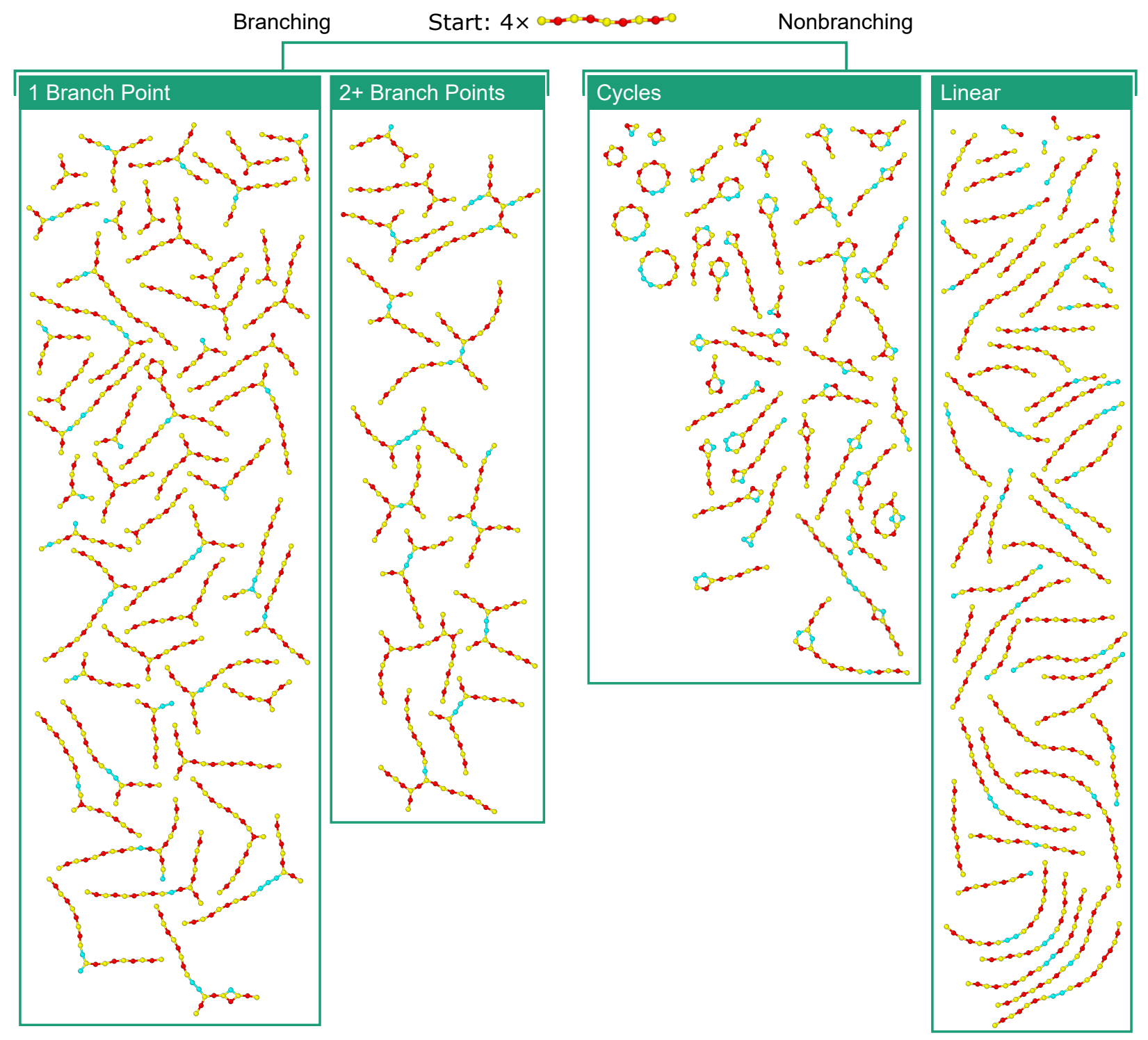

Figure 6: 2D representations of the 160 topologically unique backbones found among the 1731 backbones present at the end of the 400 independent 10 ps QMD cascade simulations. Atoms are respectively colored yellow, red, and cyan for silicon, oxygen, and carbon.

bones can be distinguished based on whether they exhibit one or more branch points. Here we take a branch point to be an atom center that joins three or more terminated backbone segments. In nearly all cases, these branch points are carbon or silicon atoms, although a few over-coordinated oxygen centers are also present. Nonbranching backbones fall into further sub-divisions. One sub-division includes completely linear backbones. Another nonbranching sub-division are those that exhibit one or more cycles that either constitute the entire 
backbone, terminate it, or else join multiple segments.

Formation of branch points is perhaps the most important chemical trend that impacts mechanical properties of polymer networks, as these are needed to form chemical cross-links. Many of the predicted branched structures are qualitatively similar to those elucidated from indirect experimental evidence. ${ }^{7,11,16}$ In particular, those with one branch point fall into the class of Y-type links. Proposed cross-linking schemes involve the formation of Y-links with a silicon center bonded to three branches though Si-O bonds, forming a Y-like shape. Such silicon-centered Y-links are indeed predicted in the QMD simulations, however we also find Y-links that involve Si-C bonds and in rare instances oxygen- and carbon-centered Ylinks. Some of the backbones with two or more branch points exhibit H-links, which involve either silmethylene $\left(\mathrm{Si}-\mathrm{CH}_{2}-\mathrm{Si}\right)$ or silethylene $\left(\mathrm{Si}_{-}-\mathrm{CH}_{2}-\mathrm{CH}_{2}-\mathrm{Si}\right)$ bridges and form an $\mathrm{H}$-like shape. Similar to the Y-links, experimentally deduced silmethylene and silethylene H-links are among those predicted in our QMD simulations. However, we also find siloxane bridges (Si-O) and more complicated carbon bridges such as propyl linkers. Some structures exhibit multiple branch points that are so close together that one cannot cleanly distinguish whether they are Y-or H-type. Substantially more Y-links are found than H-links, which is consistent with experiments. ${ }^{16}$

Not all network-altering reactions result in the creation of branch points. Many of these reactions produce linear backbones. Three local structural motifs manifest for all excitation types that can be identified among the linear backbones, and in some cases within a single backbone. These include siloxane (Si-O) segments of various lengths, the formation of carbon bridges between terminal ends of siloxane segments, and the formation of carbon termini. Linear siloxane backbone segments with fewer than nine atoms (the starting backbone size) indicate that one or more $\mathrm{Si}-\mathrm{O}$ chain-scissioning reactions took place. In some cases, these segments are joined together by carbon linkages or are terminated by carbon atoms. One interpretation of large linear backbones containing small siloxane segments is that they result from "healed" chain scissions. That is, some (if not most) chain scission reac- 
tions produce radical or ionic termini that rapidly react on a $<10$ ps timescale to recombine with other part of the polymer network in their immediate vicinity. These structures justify similar "end-linking" reactions in probabilistic models for network rearrangements through radiation damage. ${ }^{17}$ In contrast, small linear backbones identified in the graph analysis reflect a "persistent" chain scission. In rare instances, direct excitation of the backbone yields products with $\mathrm{Si}-\mathrm{Si}$ and $\mathrm{O}-\mathrm{O}$ bonds; formation of $\mathrm{Si}-\mathrm{Si}$ and $\mathrm{O}-\mathrm{O}$ bonds was not observed in simulations that excite carbon or hydrogen atoms. As Si-Si bonds are observed to form in radiolysis experiments, ${ }^{11}$ this could indicate that main-chain excitations are responsible for their production.

The last broad class of radiation-damaged backbones identified were those containing one or more cycles. Cyclization reactions are an anticipated route for chemical degradation of silicones under exposure to radiation, thermal stress, and from acidic/basic roaming gaseous contaminants. Some of those cyclic products predicted here, such the three-monomer cyclosiloxane hexamethylcyclotrisiloxane (commonly referred to as D3), are well-known degradation products. ${ }^{9}$ Nearly all of the predicted cycles involve carbon, indicating that typical chain-backbiting reactions-which involve an attack by a silicon radical on oxygen atoms-are not the dominate route to cycle formation. Since carbon is initially only in side groups, and given that carbon-containing cycles form for all four excitation types, the backbone set indicates that attacks between the main chains and side groups are relatively common. Cycle formation also occurs in sequence with other reactions, such as those forming branch points or that incorporate carbon into linear backbone segments.

\subsection{Excitation-Specific Damage Characteristics}

The local chemical environment for a PKA event as well as the chemical identity of the recoil atom are anticipated to play a role in determining radiation damage in molecular materials. Here, we combine analysis approaches to quantify excitation-specific radiation damage characteristics that have a significant bearing on the mechanical properties of polymers. 


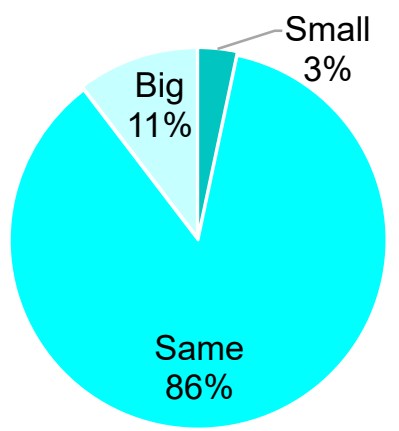

Excite C

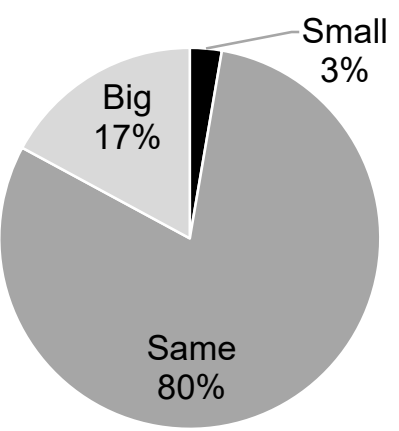

Excite $\mathrm{H}$

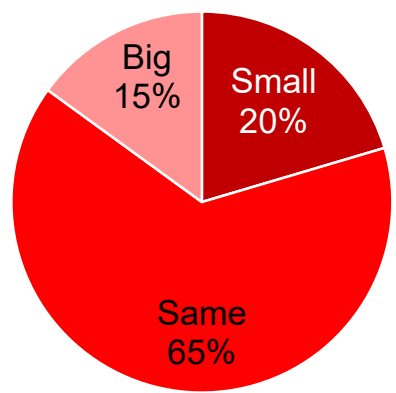

Excite O

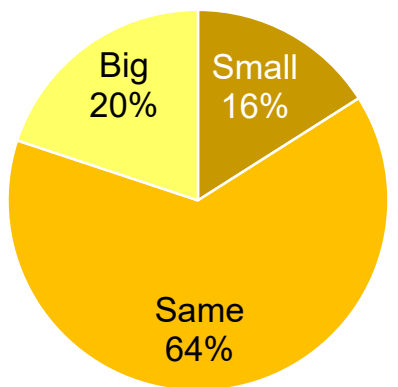

Excite Si

Figure 7: Relative populations of atoms found in backbones of three different size classes determined for each excitation type at the end of the cascade simulations. The initial backbone size is $N=9$ atoms and final backbones are considered to be smaller than the starting size if $N \leq 7$, the same within a monomer repeat unit if $8 \leq N \leq 10$, and bigger if $N \geq 11$. Populations are reported as a percent of the final total backbone atom count in each ensemble of 100 simulations. Each ensemble starts with 3600 atoms in backbones and exciting C, $\mathrm{H}, \mathrm{O}$, and Si atoms respectively lead to 3692, 3664, 3600, and 3612 atoms in backbones.

Molecular weight plays a key role in determining mechanical properties of polymer melts, with an increase in molecular weight being positively correlated with an increase in viscosity. ${ }^{10,80}$ The closest physical analog to the initial configuration in our QMD simulations is that of a low molecular weight melt of linear chains. Figure 7 shows the relative populations of atoms in different sized backbones following primary radiation damage for the four excitation types, which corresponds to time $t=10 \mathrm{ps}$ in Figure 5. For example, exciting carbon atoms leads to a net increase in the number of atoms in backbones (from 3600 to 3692). Of those 3692 atoms, $123(3.3 \%)$ are in small backbones, 3186 (86.3\%) are in same-sized backbones, and $383(10.4 \%)$ are in large backbones, with small and large backbones as defined in the Fig. 7 caption.

It is clear from this integrated view that exciting side group atoms ( $\mathrm{C}$ and $\mathrm{H}$ ) leads to substantial "uptake" of atoms into large backbones. Main chain excitation results in fewer atoms remaining in same-sized backbones. However, the ratio of atoms in big and small backbones is more finely balanced. Exciting oxygen atoms leads to a larger share of atoms in small backbones compared to large ones, and those ratios are nearly exactly reversed for exciting silicon atoms. The ranked effectiveness of each excitation type at inducing a net 
Table 2: Populations of final backbone product categories defined in Figure 6 expressed as a percent of total number of backbones for each ensemble

\begin{tabular}{crrrr}
\hline \hline Backbone Type & Excite C & Excite H & Excite O & Excite Si \\
\hline 1 Branch Point & 8.2 & 3.3 & 7.2 & 8.6 \\
2+ Branch Points & 1.0 & 1.8 & 0.2 & 1.3 \\
Nonbranching Cycles & 2.2 & 4.1 & 3.1 & 4.6 \\
Nonbranching Linear & 88.6 & 90.8 & 89.5 & 85.5 \\
\hline \hline
\end{tabular}

increase in backbone size goes as hydrogen, carbon, silicon, with hydrogen being the most effective. Oxygen is the only recoil atom type that leads to an average decrease in chain size.

Final populations of the different backbone types defined in Figure 6 are collected for each PKA excitation type in Table 2. It is immediately apparent that most of the backbones are nonbranching linear structures, comprising between $85.5 \%$ and $90.8 \%$ of the final backbones. This is at least partially because the four initial backbones in the simulation cell are all linear and not all backbones undergo alteration following a PKA event. Exciting the heavy atoms $(\mathrm{C}, \mathrm{O}$, and $\mathrm{Si}$ ) leads to more branched structures than exciting hydrogen. It is also significantly more likely for a backbone to exhibit a single branch point than multiple branch points, especially when heavy atoms are excited. In all cases, it is more likely to form branched structures than ones with cycles.

While the categorization scheme in Figure 6 provides a convenient view of network changes in this specific system starting with four DMPS chains, isolating changes in local topology allows for translating these results to other starting configurations. Figure 8 shows expected changes in backbone composition and topological features for a typical PKA event of each type. Focusing first on the top panel, it is evident that each excitation type leads to a distinct change in backbone atom content, both in terms of total number of atoms as well as the specific element types. All excitation types lead to a net decrease in oxygen content and a net increase in carbon content. Silicon is only lost from the backbones if silicon atoms are directly excited. For carbon and hydrogen exictations, the increase in carbon content is much larger than the decrease in oxygen content, leading to a relatively large net increase 

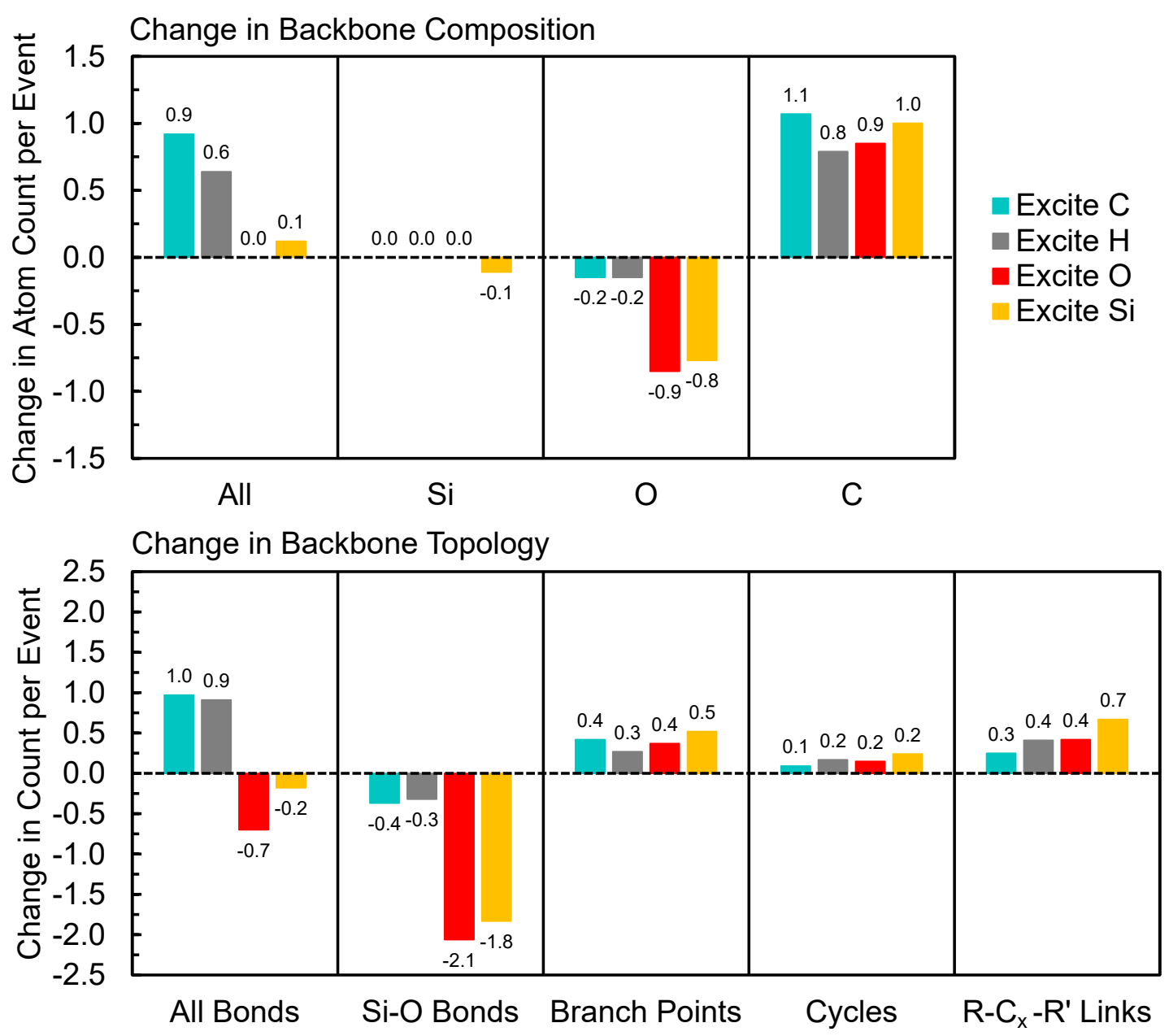

Figure 8: Ensemble-average changes in PDMS backbone composition (top panel) and topology (bottom panel) resulting from the four different excitation types. Topological features were inferred from the library of backbone structures shown in Figure 6.

in total backbone atom count. Exciting silicon and oxygen atoms in the backbone has an $\approx 90 \%$ chance for loss of one backbone atom per event which is almost exactly compensated for by carbon uptake.

Backbone topological features undergo substantial changes from a typical PKA event. As was previously identified in Figure 4(a), there is a net increase in backbone bonds for carbon and hydrogen exictations and a net decrease in backbone bonds for silicon and oxygen. It is interesting to see that all four excitation types lead to a net loss of Si-O bonds through chain scissions and other chemical reactions. Nearly two Si-O bonds are broken on average with silicon and oxygen excitations, which is perhaps to be expected. Each oxygen initially has 
two Si-O bonds and three of the five silicon atoms likewise have two Si-O bonds, with the other two terminating the backbone. The slightly larger net loss of Si-O bonds for oxygen excitations compared to silicon may be partly due to this initial difference.

More complicated topological features are formed that were identified by the isomorphism analysis used to generate the backbone library captured in Figure 6. These include branch points, cycles, and carbon linkers, which we denote as $\mathrm{R}-\mathrm{C}_{x}-\mathrm{R}$ '. Connections with multiple carbon atoms (e.g., silethylene) are considered to a single linker, and the category does not distinguish between $\mathrm{Y}$ - and H-type linkers between branch points and linkers that join terminal ends of siloxane segments. The four excitation types have a somewhat similar probability to form branch points and range from a $27 \%$ chance in the case of hydrogen to a $52 \%$ chance in the case of silicon. Probabilities for forming branch points are a key component of higher level models that capture scission and cross linking of polymer networks ${ }^{17}$ and are a natural point of connection for upscaling QMD results to inform macroscale models.

\section{Conclusions}

We develop here an in silico approach to predict and quantify fundamental chemical changes to polymer networks and apply this to study ionizing radiation damage of polydimethylsiloxane (PDMS), the primary constituent of silicones. Our approach combines semiempirical quantum-based molecular dynamics (QMD) sampling with a tailored graph-based molecular structure recognition tool to isolate key changes to network topology with a significant bearing on mechanical properties. Radiation damage is modeled starting from primary knock-on atom (PKA) events, and the consequences of exciting each element type in PDMS (Si, O, $\mathrm{C}$, and $\mathrm{H}$ ) are examined in detail. The density functional tight-binding (DFTB) method is used to perform hundreds of independent QMD simulations of radiation-induced reaction cascades, generating ns of trajectory data from which to extract statistics.

Graph-based analysis tools are developed to automate the identification of polymer back- 
bone structures and assemble a complete library of all topologically distinct backbones sampled in reactive MD simulations. These analyses reveal a wide diversity of changes to siloxane polymer networks following irradiation, including the formation of branch points, carbon linkages, cycles, and bond scissions. Many of the basic topological motifs identified are consistent with experimentally deduced siloxane radiolysis chemistry, but our results indicate there is a much higher degree of combinatorial complexity in this system. Characteristics for damage ensuing from initial radiation coupling to side group $(\mathrm{C}$ and $\mathrm{H})$ and main chain (Si and $\mathrm{O}$ ) atoms are extracted, with most couplings leading to a net increase in backbone size through cross-linking and recombination reactions. While exciting $\mathrm{H}$ and $\mathrm{C}$ atoms in the side groups was anticipated to lead to formation of carbon-based backbone linkages, we find that all four excitation types are effective in driving carbon uptake into the network. Statistics obtained through our approach yield probabilities for the fundamental reactions involved in the formation of network cross links and main chain scissions in irradiated PDMS.

Combining efficient semiempirical QMD simulations with graph-based molecular recognition tools enables high-throughput sampling of dynamically evolving chemistry. Our approach has a decided advantage in that it uses non-degenerate molecule descriptors, enabling the reliable identification of all chemically unique structures sampled in a system. This is of particular relevance to situations requiring more than a simplified or reduced-order description of the underlying chemistry or in cases where important chemical trends cannot be anticipated a priori. The general analysis framework presented here is readily extendable to modeling chemical degradation of other polymers and molecular materials and provides a basis for future quantum-informed multiscale modeling of radiation damage.

\section{Acknowledgement}

This work was performed under the auspices of the U.S. Department of Energy by Lawrence Livermore National Laboratory under Contract DE-AC52-07NA27344. Released under doc- 
ument number LLNL-JRNL-814324-DRAFT.

\section{References}

(1) Bruck, S. D.; Mueller, E. P. Radiation sterilization of polymeric implant materials. $J$. Biomed. Mater. Res. Appl. Biomater. 1988, 22, 133-144.

(2) Williams, I. R.; Mayor, M. B.; Collier, J. P. The Impact of Sterilization Method on Wear in Knee Arthroplasty. Clin. Orthop. Relat. Res. 1998, 356, 170-180.

(3) Almeida, J. C.; Lancastre, J.; Fernandes, M. H. V.; Margaça, F. M. A.; Ferreira, L.; Salvado, I. M. M. Evaluating structural and microstructural changes of PDMS-SiO2 hybrid materials after sterilization by gamma irradiation. Mater. Sci. Eng. C 2015, $48,354-358$.

(4) Chinn, S.; DeTeresa, S.; Sawvel, A.; Shields, A.; Balazs, B.; Maxwell, R. S. Chemical origins of permanent set in a peroxide cured filled silicone elastomer - tensile and $1 \mathrm{H}$ NMR analysis. Polym. Degrad. Stab. 2006, 91, 555 - 564.

(5) Maiti, A.; Gee, R.; Weisgraber, T.; Chinn, S.; Maxwell, R. Constitutive modeling of radiation effects on the permanent set in a silicone elastomer. Polym. Degrad. Stab. 2008, 93, $2226-2229$.

(6) Labouriau, A.; Robison, T.; Geller, D.; Cady, C.; Pacheco, A.; Stull, J.; Dumont, J. H. Coupled aging effects in nanofiber-reinforced siloxane foams. Polym. Degrad. Stab. 2018, 149, $19-27$.

(7) Palsule, A. S.; Clarson, S. J.; Widenhouse, C. W. Gamma Irradiation of Silicones. J. Inorg. Organomet. P. 2008, 18, 207-221.

(8) Hill, D. J. T.; Whittaker, A. K. Encyclopedia of Polymer Science and Technology; American Cancer Society, 2016; pp 1-58. 
(9) Camino, G.; Lomakin, S.; Lageard, M. Thermal polydimethylsiloxane degradation. Part 2. The degradation mechanisms. Polymer 2002, 43, $2011-2015$.

(10) Charlesby, A.; Freeth, F. A. Changes in silicone polymeric fluids due to high-energy radiation. Proc. R. Soc. Lond. A 1955, 230, 120-135.

(11) Miller, A. A. Radiation Chemistry of Polydimethylsiloxane.1 I. Crosslinking and Gas Yields. J. Am. Chem. Soc. 1960, 82, 3519-3523.

(12) Kornacka, E.; Kozakiewicz, J.; Legocka, I.; Przybylski, J.; Przybytniak, G.; Sadło, J. Radical processes induced in poly(siloxaneurethaneureas) by ionising radiation. Polym. Degrad. Stab. 2006, 91, $2182-2188$.

(13) Labouriau, A.; Cady, C.; Gill, J.; Taylor, D.; Zocco, A.; Stull, J.; Henderson, K.; Wrobleski, D. The effects of gamma irradiation on RTV polysiloxane foams. Polym. Degrad. Stab. 2015, 117, $75-83$.

(14) LaVerne, J. A.; Tratnik, N. A.; Sasgen, A. Gas production in the radiolysis of Poly(dimethysiloxanes). Radiat. Phys. Chem. 2018, 142, 50 - 53, Ionizing Radiations and Polymers, IRaP-2016.

(15) Wang, P.-C.; Yang, N.; Liu, D.; Qin, Z.-M.; An, Y.; Chen, H.-B. Coupling effects of gamma irradiation and absorbed moisture on silicone foam. Mater. Des. 2020, 195, 108998.

(16) Hill, D. J.; Preston, C. M.; Salisbury, D. J.; Whittaker, A. K. Molecular weight changes and scission and crosslinking in poly(dimethyl siloxane) on gamma radiolysis. Radiat. Phys. Chem. 2001, 62, $11-17$.

(17) Dinh, L. N.; Mayer, B. P.; Maiti, A.; Chinn, S. C.; Maxwell, R. S. Molecular weight distributions of irradiated siloxane-based elastomers: A complementary study by statis- 
tical modeling and multiple quantum nuclear magnetic resonance. J. Appl. Phys. 2011, 109, 094905.

(18) Pankratova, L. N.; Saenko, E. V.; Bugaenko, V. L.; Makhlyarchuk, V. V. Kinetics of decay of methyl radicals in irradiated dimethylsilanediol and polymethylsiloxanes. Mendeleev Commun. 2011, 21, 157 - 159.

(19) Lewicki, J. P.; Maxwell, R. S.; Mayer, B. P.; Maiti, A.; Harley, S. J. In Concise Encyclopedia of High Performance Silicones; Tiwari, A., Soucek, M. D., Eds.; Wiley, 2014; Chapter 11, pp 151-176.

(20) Rottach, D. R.; Curro, J. G.; Grest, G. S.; Thompson, A. P. Effect of strain history on stress and permanent set in cross-linking networks: A molecular dynamics study. Macromol. 2004, 37, 5468-5473.

(21) Chenoweth, K.; Cheung, S.; van Duin, A. C. T.; Goddard, W. A.; Kober, E. M. Simulations on the thermal decomposition of a poly(dimethylsiloxane) polymer using the ReaxFF reactive force field. J. Am. Chem. Soc. 2005, 127, 7192-7202.

(22) Lupton, E. M.; Achenbach, F.; Weis, J.; Bräuchle, C.; Frank, I. Molecular origins of adhesive failure: Siloxane elastomers pulled from a silica surface. Phys. Rev. B: Condens. Matter Mater. Phys. 2007, 76, 125420.

(23) Lupton, E. M.; Achenbach, F.; Weis, J.; Bräuchle, C.; Frank, I. Origins of material failure in siloxane elastomers from first principles. ChemPhysChem 10, 119-123.

(24) Deetz, J. D.; Faller, R. Parallel Optimization of a Reactive Force Field for Polycondensation of Alkoxysilanes. J. Phys. Chem. B 2014, 118, 10966-10978.

(25) Polvi, J.; Luukkonen, P.; Nordlund, K.; Järvi, T. T.; Kemper, T. W.; Sinnott, S. B. Primary Radiation Defect Production in Polyethylene and Cellulose. J. Phys. Chem. B 2012, 116, 13932-13938. 
(26) Polvi, J.; Nordlund, K. Low-energy irradiation effects in cellulose. J. Appl. Phys. 2014, $115,023521$.

(27) Koo, B.; Subramanian, N.; Chattopadhyay, A. Molecular dynamics study of brittle fracture in epoxy-based thermoset polymer. Compos. B Eng. 2016, 95, 433 - 439.

(28) Kroonblawd, M. P.; Goldman, N.; Lewicki, J. P. Chemical degradation pathways in siloxane polymers following phenyl excitations. J. Phys. Chem. B 2018, 122, 1220112210.

(29) Nordlund, K.; Zinkle, S. J.; Sand, A. E.; Granberg, F.; Averback, R. S.; Stoller, R. E.; Suzudo, T.; Malerba, L.; Banhart, F.; Weber, W. J. et al. Primary radiation damage: A review of current understanding and models. J. Nucl. Mater. 2018, 512, $450-479$.

(30) Gibson, J. B.; Goland, A. N.; Milgram, M.; Vineyard, G. H. Dynamics of Radiation Damage. Phys. Rev. 1960, 120, 1229-1253.

(31) Robinson, M. T.; Torrens, I. M. Computer simulation of atomic-displacement cascades in solids in the binary-collision approximation. Phys. Rev. B: Condens. Matter Mater. Phys. 1974, 9, 5008-5024.

(32) de la Rubia, T. D.; Averback, R. S.; Benedek, R.; King, W. E. Role of thermal spikes in energetic displacement cascades. Phys. Rev. Lett. 1987, 59, 1930-1933.

(33) Wood, M. A.; Cusentino, M. A.; Wirth, B. D.; Thompson, A. P. Data-driven material models for atomistic simulation. Phys. Rev. B: Condens. Matter Mater. Phys. 2019, 99, 184305.

(34) Stoller, R. In Comprehensive Nuclear Materials; Konings, R. J., Ed.; Elsevier: Oxford, 2012; pp $293-332$.

(35) Ruault, M. O.; Chaumont, J.; Penisson, J. M.; Bourret, A. High resolution and in situ investigation of defects in Bi-irradiated Si. Philos. Mag. A 1984, 50, 667-675. 
(36) Holland, O.; Narayan, J.; Fathy, D. Ion beam processes in Si. Nucl. Instrum. Methods $B$ 1985, 7-8, $243-250$.

(37) Williams, J. Ion implantation of semiconductors. Mater. Sci. Eng. A 1998, 253, 8 15.

(38) Lieb, K.-P.; Keinonen, J. Luminescence of ion-irradiated $\alpha$-quartz. Contemp. Phys. 2006, 4\%, 305-331.

(39) Banhart, F.; Kotakoski, J.; Krasheninnikov, A. V. Structural Defects in Graphene. ACS Nano 2011, 5, 26-41.

(40) Honeycutt, J. D.; Andersen, H. C. Molecular dynamics study of melting and freezing of small Lennard-Jones clusters. J. Phys. Chem. 1987, 91, 4950-4963.

(41) Chau, P. L.; Hardwick, A. J. A new order parameter for tetrahedral configurations. Mol. Phys. 1998, 93, 511.

(42) Ackland, G. J.; Jones, A. P. Applications of local crystal structure measures in experiment and simulation. Phys. Rev. B: Condens. Matter Mater. Phys. 2006, 73, 054104.

(43) Larsen, P. M.; Schmidt, S.; Schiøtz, J. Robust structural identification via polyhedral template matching. Model. Simul. Mater. Sci. Eng. 2016, 24, 055007.

(44) Akutsu, T.; Nagamochi, H. Comparison and enumeration of chemical graphsS. Comput. Struct. Biotec. 2013, 5, e201302004.

(45) Karypis, G.; Kumar, V. A Fast and High Quality Multilevel Scheme for Partitioning Irregular Graphs. SIAM J. Sci. Comput. 1998, 20, 359-392.

(46) Hagberg, A. A.; Schult, D. A.; Swart, P. J. Exploring network structure, dynamics, and function using NetworkX. Proceedings of the 7th Python in Science Conference. Pasadena, CA USA, 2008; pp 11 - 15, NetworkX is available at https://networkx.github.io (accessed Aug 27, 2019). 
(47) Niklasson, A. M. N.; Mniszewski, S. M.; Negre, C. F. A.; Cawkwell, M. J.; Swart, P. J.; Mohd-Yusof, J.; Germann, T. C.; Wall, M. E.; Bock, N.; Rubensson, E. H. et al. Graphbased linear scaling electronic structure theory. J. Chem. Phys. 2016, 144, 234101.

(48) Ghale, P.; Kroonblawd, M. P.; Mniszewski, S.; Negre, C. F. A.; Pavel, R.; Pino, S.; Sardeshmukh, V.; Shi, G.; Hahn, G. Task-based Parallel Computation of the Density Matrix in Quantum-based Molecular Dynamics using Graph Partitioning. SIAM J. Sci. Comput. 2017, 39, C466-C480.

(49) Karnes, J. J.; Benjamin, I. Miscibility at the immiscible liquid/liquid interface: A molecular dynamics study of thermodynamics and mechanism. J. Chem. Phys. 2018, $148,034707$.

(50) Kroonblawd, M. P.; Goldman, N.; Lewicki, J. P. Anisotropic Hydrolysis Susceptibility in Deformed Polydimethylsiloxanes. J. Phys. Chem. B 2019, 123, 7926-7935.

(51) Zhou, T.; Martinez-Baez, E.; Schenter, G.; Clark, A. E. PageRank as a collective variable to study complex chemical transformations and their energy landscapes. $J$. Chem. Phys. 2019, 150, 134102.

(52) Kroonblawd, M. P.; Fried, L. E. High Explosive Ignition through Chemically Activated Nanoscale Shear Bands. Phys. Rev. Lett. 2020, 124, 206002.

(53) Kohn, W.; Sham, L. J. Self-consistent equations including exchange and correlation effects. Phys. Rev. 1965, 140, A1133-A1138.

(54) Elstner, M.; Porezag, D.; Jungnickel, G.; Elsner, J.; Haugk, M.; Frauenheim, T.; Suhai, S.; Seifert, G. Self-consistent-charge density-functional tight-binding method for simulations of complex materials properties. Phys. Rev. B: Condens. Matter Mater. Phys. 1998, 58, $7260-7268$. 
(55) Koskinen, P.; Mäkinen, V. Density-functional tight-binding for beginners. Comput. Mater. Sci. 2009, 47, $237-253$.

(56) Kroonblawd, M. P.; Lindsey, R. K.; Goldman, N. Synthesis of functionalized nitrogencontaining polycyclic aromatic hydrocarbons and other prebiotic compounds in impacting glycine solutions. Chem. Sci. 2019, 10, 6091-6098.

(57) Martínez, E.; Perriot, R.; Kober, E. M.; Bowlan, P.; Powell, M.; McGrane, S.; Cawkwell, M. J. Parallel replica dynamics simulations of reactions in shock compressed liquid benzene. J. Chem. Phys. 2019, 150, 244108.

(58) Cawkwell, M. J.; Manner, V. W. Ranking the Drop-Weight Impact Sensitivity of Common Explosives Using Arrhenius Chemical Rates Computed from Quantum Molecular Dynamics Simulations. J. Phys. Chem. A 2020, 124, 74-81.

(59) Kroonblawd, M.; Goldman, N. Carbon in Earth's Interior; American Geophysical Union (AGU), 2020; Chapter 23, pp 271-283.

(60) Perriot, R.; Cawkwell, M. J.; Martinez, E.; McGrane, S. D. Reaction Rates in Nitromethane under High Pressure from Density Functional Tight Binding Molecular Dynamics Simulations. J. Phys. Chem. A 2020, 124, 3314-3328.

(61) Arkles, B. Look what you can make out of silicones (biomedical applications of silicones). 1983, 13, 542-555.

(62) Aradi, B.; Hourahine, B.; Frauenheim, T. DFTB +, a sparse matrix-based implementation of the DFTB method. J. Phys. Chem. A 2007, 111, 5678-5684, DFTB + is available at https://www.dftb-plus.info (accessed Aug 27, 2019).

(63) Slater, J. C.; Koster, G. F. Simplified LCAO method for the periodic potential problem. Phys. Rev. 1954, 94, $1498-1524$. 
(64) Rappe, A. K.; Casewit, C. J.; Colwell, K. S.; Goddard III, W. A.; Skiff, W. M. UFF, a full periodic table force field for molecular mechanics and molecular dynamics simulations. J. Am. Chem. Soc. 1992, 114, 10024-10035.

(65) Mermin, N. D. Thermal properties of the inhomogeneous electron gas. Phys. Rev. 1965, 137, A1441 - A1443.

(66) Niklasson, A. M. N.; Tymczak, C. J.; Challacombe, M. Time-reversible BornOppenheimer molecular dynamics. Phys. Rev. Lett. 2006, 97, 123001.

(67) Niklasson, A. M. N. Extended Born-Oppenheimer molecular dynamics. Phys. Rev. Lett. 2008, 100, 123004.

(68) Niklasson, A. M. N.; Steneteg, P.; Odell, A.; Bock, N.; Challacombe, M.; Tymczak, C. J.; Holmström, E.; Zheng, G.; Weber, V. Extended Lagrangian Born-Oppenheimer molecular dynamics with dissipation. J. Chem. Phys. 2009, 130, 214109.

(69) Zheng, G.; Niklasson, A. M. N.; Karplus, M. Lagrangian formulation with dissipation of Born-Oppenheimer molecular dynamics using the density-functional tight-binding method. J. Chem. Phys. 2011, 135, 044122.

(70) Grigoras, S.; Qian, C.; Crowder, C.; Harkness, B.; Mita, I. Structure elucidation of crystalline poly(diphenylsiloxane). Macromol. 1995, 28, 7370-7375.

(71) Tosaka, M.; Tashiro, K. Crystal polymorphism and structure models of Poly(dimethylsiloxane). Polymer 2018, 153, $507-520$.

(72) Plimpton, S. Fast parallel algorithms for short-range molecular dynamics. J. Comput. Phys. 1995, 117, 1 - 19, LAMMPS is available at http://lammps.sandia.gov (accessed Aug 27, 2019). 
(73) Nosé, S. A unified formulation of the constant temperature molecular dynamics methods. J. Chem. Phys. 1984, 81, $511-519$.

(74) Hoover, W. G. Canonical dynamics: Equilibrium phase-space distributions. Phys. Rev. A: At., Mol., Opt. Phys. 1985, 31, 1695 - 1697.

(75) Kroonblawd, M. P.; Sewell, T. D. Anisotropic Relaxation of Idealized Hot Spots in Crystalline 1,3,5-Triamino-2,4,6-trinitrobenzene (TATB). J. Phys. Chem. C 2016, 120, $17214-17223$.

(76) Cordella, L. P.; Foggia, P.; Sansone, C.; Vento, M. An improved algorithm for matching large graphs. In: 3rd IAPR-TC15 Workshop on Graph-based Representations in Pattern Recognition, Cuen. 2001; pp 149-159.

(77) Stukowski, A. Visualization and analysis of atomistic simulation data with OVITO the open visualization tool. Model. Simul. Mater. Sci. Eng. 2010, 18, 015012, OVITO is available at https://www.ovito.org (accessed Aug 27, 2019).

(78) Weininger, D. SMILES, a chemical language and information system. 1. Introduction to methodology and encoding rules. J. Chem. Inf. Comput. Sci. 1988, 28, 31-36.

(79) Weininger, D.; Weininger, A.; Weininger, J. L. SMILES. 2. Algorithm for generation of unique SMILES notation. J. Chem. Inf. Comput. Sci. 1989, 29, 97-101.

(80) Fox, T. G.; Flory, P. J. Second-Order Transition Temperatures and Related Properties of Polystyrene. I. Influence of Molecular Weight. J. Appl. Phys. 1950, 21, 581-591. 


\section{Graphical TOC Entry}

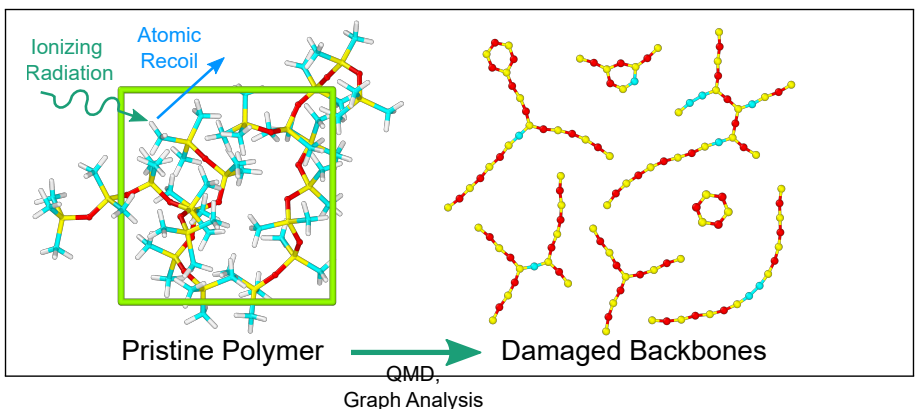

